$\tau_{w} \quad=t_{0} /\left\{\left(\varepsilon \rho_{g} C_{p g}+\gamma C_{p s}\right) r / 2 h_{0}\right\}$

$\xi \quad=$ defined by Eq. (5)

Literature Cited

1) Chihara, K. and M. Suzuki: J. Chem. Eng. Japan, 16, 53 (1983).

2) Crank, J.: "The Mathematics of Diffusion," Oxford (1975).

3) Mitchell, J. E. and L. H. Shendalman: AIChE Symp. Ser., 69, No. 134, 25 (1973)
4) Mizushina, T. et al.: "Heat Transfer and Heat Exchanger," in Kagaku Kogaku Binran, pp. 255-385, Maruzen, Tokyo (1978).

5) Nakao, S. and M. Suzuki: J. Chem. Eng. Japan, 16, 114 (1983).

6) Skarstrom, C. W.: "Recent Developments in Separation Science," Vol. 2, pp. 95-106, CRC Press, Cleveland, Ohio (1972)

7) Shendalman, L. H. and J. E. Mitchell: Chem. Eng. Sci., 27, $1449(1972)$

\title{
TURBULENT COAGULATION OF PARTICLES DISPERSED IN A VISCOUS FLUID
}

\author{
KO HIGASHITANI, KIYOYUKI YAMAUCHI, YOSHIZO MATSUNO \\ AND GIJIRO HOSOKAWA \\ Department of Environmental Engineering, Kyushu Institute of Technology, Kitakyushu 804
}

\begin{abstract}
A kinetic equation for the turbulent coagulation of particles in a viscous fluid in which the hydrodynamic interaction between colliding particles is taken into account is proposed. The theoretical prediction of the timedependent behavior of particle concentration is compared with results of experiments in which latex particles in $\mathrm{KCl}$ solutions are coagulated in a stirred tank. It is found that the effect of the hydrodynamic interaction is not negligible, and that changes of particle concentration under various experimental conditions are quantitatively predicted by the present theory. On the other hand, the Saffman-Turner theory is found to overestimate the coagulation rate considerably and to yield a systematic error in the dependence of coagulation rate on the dissipation energy.
\end{abstract}

\section{Introduction}

The coagulation of colloidal particles is found in many natural phenomena and industrial processes. In most situations the coagulation is caused by the flow of turbulence, where particles collide because of their relative motion induced by the velocity field of the medium. If there exists no repulsive surface force between particles, every collision leads to coagulation. This process, called rapid coagulation, in a turbulent viscous fluid is treated in this paper.

A theoretical treatment of turbulent coagulation was carried out approximately by Camp and Stein, ${ }^{2)}$ assuming that the collision mechanism of particles is analogous to that of the shear coagulation proposed by Smoluchowski. ${ }^{12)}$ Levich $^{7}$ also derived a kinetic equation of turbulent coagulation, assuming that the problem of coagulation of particles transported by turbulent eddies is reduced to a diffusion problem characterized by a turbulent diffusion coefficient. A

Received September 27, 1982. Correspondence concerning this article should be addressed to Ko Higashitani. Y. Matsuno is at Dept. of Industrial Chemistry, Kyushu Institute of Tech., Kitakyushu 804. K. Yamauchi is now with Fuji Electric Co., Ltd., Hino 191. theory developed by Saffman and Turner ${ }^{11)}$ is somewhat more rigorous. However, it is assumed in their analysis that particles encounter each other without interacting hydrodynamically; that is, each particle behaves as though the others were not present. This theory is approximately applicable to the collision in which the effect of inertia of particles is predominant, or the interparticle attractive force acts such that it cancels the effect of the hydrodynamic interaction. But it is unlikely that the theory is applicable to the collision between sufficiently small particles dispersed in a viscous fluid. The theories described above were developed to predict the coagulation process in which particles follow the fluid motion completely. There is another mechanism of turbulent coagulation in which the particle collision is attributed to the inertia of particles. This mechanism is not treated here because it is applicable to rather large particles in a vigorous turbulence.

Experimental investigations on turbulent coagulation have been carried out for aerosols, ${ }^{1,10)}$ but no rigorous measurements for colloidal dispersions in aqueous solutions are found. 
In the present paper we propose a kinetic equation for the coagulation of particles in a viscous fluid of homogeneous isotropic turbulence in which the hydrodynamic interaction between particles is taken into account. And the theoretical prediction of the time-dependent behavior of particle concentration is compared with results of experiments in which latex particles are coagulated in a stirred tank using $\mathrm{KCl}$ solutions as the coagulant.

\section{Kinetic Equation of Turbulent Coagulation}

Consider a binary collision between particles of sizes $i$ and $j$ in a viscous fluid of homogeneous isotropic turbulence. Saffman and Turner ${ }^{11)}$ calculated the collision frequency per unit volume, $J_{T i j}^{0}$, by counting the number of particles flowing into the sphere of coagulation radius $R_{i j}\left(=a_{i}+a_{j}\right.$, where $a_{i}$ is the radius of particle of size $i$ ).

$$
J_{T i j}^{0}=1.294 R_{i j}^{3}(\varepsilon / v)^{1 / 2} n_{i} n_{j}
$$

where $\varepsilon$ is the energy dissipation per unit mass of fluid, $v$ is the kinematic viscosity of the medium and $n_{i}$ is the number concentration of particle $i$. Then the rate of change in concentration of particle $k$ is given by taking a population balance.

$$
\begin{aligned}
\frac{d n_{k}}{d t}= & \frac{1}{2} \sum_{\substack{i=1 \\
i+j=k}}^{i=k-1} 1.294 R_{i j}^{3}(\varepsilon / v)^{1 / 2} n_{i} n_{j} \\
& -\sum_{i=1}^{\infty} 1.294 R_{i k}^{3}(\varepsilon / v)^{1 / 2} n_{i} n_{k} \quad(k=1,2, \cdots, \infty)
\end{aligned}
$$

where $t$ is the time. The first term on the right-hand side represents the rate of formation of particle $k$ by coagulation of smaller particles and the second term the rate of disappearance due to coagulation of particle $k$ with others.

In deriving this equation the following assumptions are required.

(1) $R_{i j}$ is small in comparison with the smallest eddies of turbulence.

(2) Particles move with the fluid velocity.

(3) Effects of inertia and sedimentation of particles are negligible.

(4) The effect of the Brownian coagulation which proceeds simultaneously is negligible.

(5) Flocs never break up.

(6) There exists no interparticle force, and particles coagulate when they collide.

(7) The collision efficiency is unity, that is, the distortion of the particle trajectory by the hydrodynamic interaction between particles is neglected.

It is known ${ }^{7)}$ that assumptions (1), (3) and (4) are usually satisfied in the case of turbulent coagulation of aqueous colloidal dispersions. Assumption (5) is acceptable at a rather early stage of coagulation where flocs are too small to break up. However, the other assumptions will not be satisfied when Eq. (2) is applied to the coagulation of aqueous dispersions. The theory is modified as follows.

Contrary to assumption (6), there exist interparticle forces between particles in an aqueous solution. Since it is conventional to assume that the van der WaalsLondon force is the only interparticle force in rapid coagulation, the other forces are not considered here. The retarded and unretarded van der Waals-London forces whose potentials are given in the previous paper $^{5)}$ are employed in calculating the coagulation coefficient given below.

It was shown ${ }^{5)}$ that particles in a shear flow interact hydrodynamically when they are close to each other, and collide along a complicated trajectory. The collision frequency $J_{S i j}$ is given by

$$
J_{S i j}=\alpha_{S i j} J_{S i j}^{0}
$$

where $\alpha_{S i j}$ is the coagulation coefficient of shear coagulation by which the effects of the hydrodynamic interaction and the van der Waals-London force are represented, and $J_{S i j}^{0}$ is the collision frequency given by the Smoluchowski theory of shear coagulation in which assumptions (2), (6) and (7) are assumed. ${ }^{12)}$ Since the collision of particles in a turbulent fluid is also attributed to the velocity gradient of the fluid, the effect of the hydrodynamic interaction will not be negligible. Then assumption (2) is invalid and assumption (7) may yield a considerable error. Since the definite flow field is not known in the case of turbulence, the hydrodynamic interaction cannot be evaluated exactly. However, it is plausible to expect that a flow field similar to the shear flow is found in the eddies of turbulence microscopically. Hence multiplying Eq. (1) by $\alpha_{S i j}$ suggests a first approximation for the collision frequency of turbulent coagulation. Since it was shown ${ }^{5)}$ that $\alpha_{S i j}$ is replaced by $\alpha_{S 11}$ of initial particles without any serious error, $\alpha_{S 11}$ may be regarded as the coagulation coefficient of turbulent coagulation $\alpha_{T 11}\left(\equiv \alpha_{T}\right)$. Then Eq. (2) is rewritten in dimensionless form as

$$
\begin{array}{r}
\frac{d n_{k}^{*}}{d t^{*}}=\frac{1}{2} \sum_{\substack{i=1 \\
i+j=k}}^{i=k-1}\left(\frac{R_{i j}}{a_{1}}\right)^{3} n_{i}^{*} n_{j}^{*}-\sum_{i=1}^{\infty}\left(\frac{R_{i k}}{a_{1}}\right)^{3} n_{i}^{*} n_{k} * \\
(k=1,2, \cdots, \infty)
\end{array}
$$

where $t^{*}=1.294 \alpha_{T}(\varepsilon / v)^{1 / 2} a_{1}{ }^{3} N_{0} t, n_{i}{ }^{*}=n_{i} / N_{0}$ and $N_{0}$ is the initial particle concentration. It is clear that this equation is reduced to Eq. (2) when $\alpha_{T}=1$. The change of particle concentration is given by solving Eq. (4) for particles of all sizes simultaneously.

\section{Experimental}

Polystyrene latex (PSL) particles $(0.85 \mu \mathrm{m})$ and 
polyvinyltoluene latex (PVTL) particles $(2.17 \mu \mathrm{m})$ were selected as the colloidal particles for the present study. Stable dispersions of these particles in water were prepared by a method reported elsewhere. ${ }^{6)}$ It was confirmed by the Coulter Counter and electron microscope that the size distribution of particles was nearly monodispersed and their shape was spherical. The effect of sedimentation and inertia of particles was regarded as negligible because the densities of PSL and PVTL particles were 1.05 and 1.02 respectively. $\mathrm{KCl}$ solutions were employed as the electrolyte solution. To prevent cavitation, air dissolved in colloidal dispersions and $\mathrm{KCl}$ solutions was eliminated by the pressure reduction method before experiments.

The experimental apparatus is shown in Fig. 1. The colloidal dispersion and $\mathrm{KCl}$ solution were fed into a mixing cell with the same flow rate, and then into a stirred tank with 4 baffles. As soon as the tank was filled, the impeller, an 8-flat-blade paddle, was rotated. Small volumes of the dispersion was sampled periodically using syringes with a needle of $1.5 \mathrm{~mm}$ diameter and the particle concentration was determined by the Coulter Counter. The manner of sampling, the location of sampling and the deposition of particles on the tank wall were found not to influence the result. All the experiments were carried out in the rapid coagulation region, that is, in $0.75 \pm 0.1 \mathrm{~mol} / 1$ $\mathrm{KCl}$ solutions at room temperature where the effect of the interparticle repulsion force due to the electrochemical double layer was avoided. ${ }^{6)}$

Measuring the torque on the impeller $T$, the experimental value of energy dissipation $\varepsilon_{0}$ was evaluated by the following equation, where all the input energy was assumed to be dissipated in the bulk of the tank.

$$
\varepsilon_{0}=2 \pi N_{I} T / W
$$

where $N_{I}$ is the rotational impeller speed and $W$ is the mass of fluid in the tank.

\section{Experimental Results}

The change of reduced total particle concentration $N_{t} / N_{0}$ is examined for various experimental conditions. Figure 2 shows the change of $N_{t} / N_{0}$ for dispersions of $0.85 \mu \mathrm{m}$ particles of different initial concentrations. It is found that the relation $N_{t} / N_{0}$ vs. $t$ depends on $N_{0}$ and that the coagulation rate increases rapidly with $N_{0}$. The dependence of the coagulation rate on $N_{0}$ is examined in Fig. 3. We define the coagulation time $T_{c}$ as the elapsed time when $N_{t}$ is reduced to half of $N_{0}$. Then $1 / T_{c}$ may be regarded as a measure of coagulation rate. It is found that $1 / T_{c}$ is proportional to about $N_{0}{ }^{1.49}$. Figure 4 shows the change of $N_{t} / N_{0}$ of dispersions of $0.85 \mu \mathrm{m}$ particles for various impeller speeds in which $N_{0}$ is about the same.

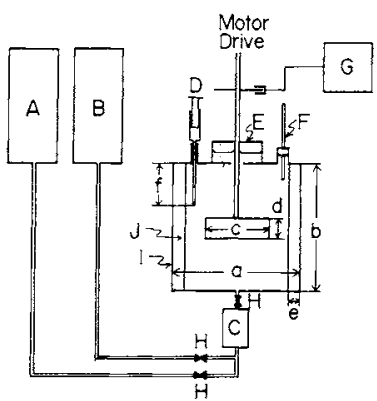

A, B Colloid, KCl reservoir ; C Mixing cell; D Sampling syringe; E Reserve tank; $F$ Thermometer; $G$ Counter: $H$ Valve ; I Tank, J Baffle

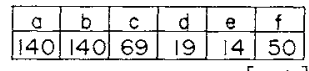

\begin{tabular}{|c|c|c|c|c|c|}
\hline 140 & 140 & 69 & 19 & 4 & 50 \\
\hline
\end{tabular}

Fig. 1. Experimental apparatus.

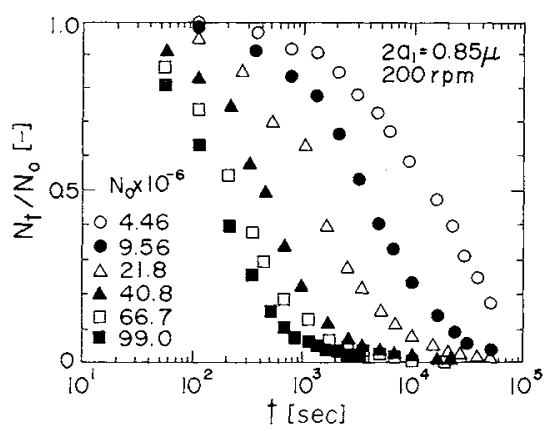

Fig. 2. Change of $N_{t} / N_{0}$ for various initial concentrations of particles.

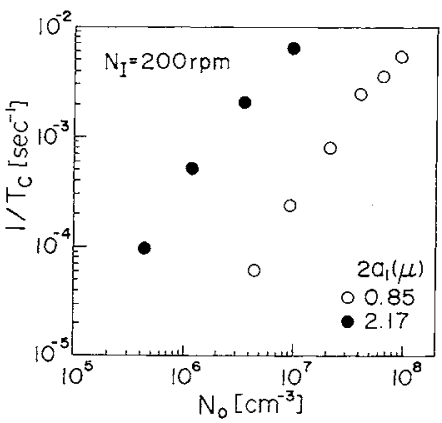

Fig. 3. Dependence of $1 / T_{c}$ on $N_{0}$.

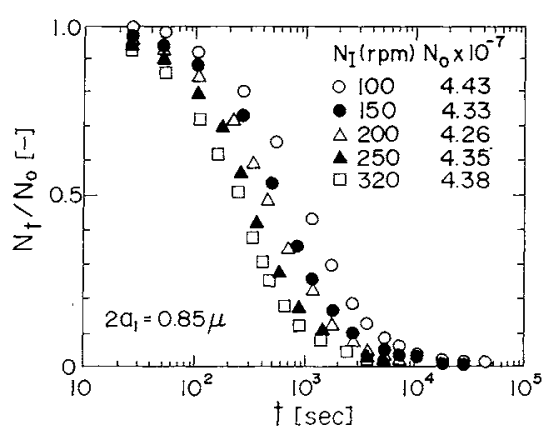

Fig. 4. Change of $N_{t} / N_{0}$ for various impeller speeds.

The dependence of $1 / T_{c}$ on $N_{I}$ is examined in Fig. 5. These figures indicate that relations $N_{t} / N_{0}$ vs. $t$ are similar in shape and that $1 / T_{c}$ is proportional to about 


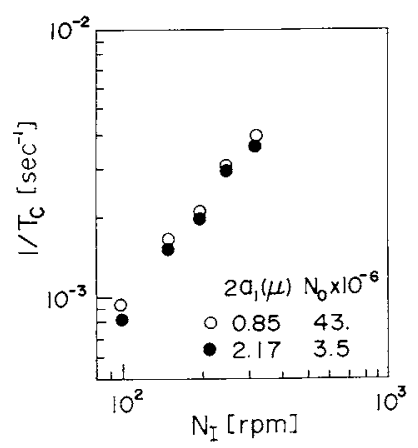

Fig. 5. Dependence of $1 / T_{c}$ on $N_{I}$.

$N_{I}^{1.27}$. Similar features are also found for dispersions of $2.17 \mu \mathrm{m}$ particles. To examine the effect of particle size, the change of $N_{t} / N_{0}$ is plotted in Fig. 6 for dispersions in which particles are of about the same concentration but differ in size. It is clear that particle size has a profound influence on the coagulation rate.

When coagulation proceeds for a sufficiently long time, $N_{t} / N_{0}$ is reduced to a constant value where the rate of coagulation is equal to the rate of floc breakup. Since this equilibrium value is quite small in this experiment, it is plausible to assume that the effect of floc break-up is negligible. This assumption is confirmed by a separate experiment in which it is examined whether flocs are redispersed by strong agitation.

\section{Discussion}

An isotropic and homogeneous turbulence is assumed in the theory. It was shown experimentally ${ }^{93}$ that the assumption is acceptable as a first approximation for flows in a stirred tank, although the turbulence in the impeller stream deviates from isotropy and homogeneity. Hence it is plausible to compare the theoretical prediction with the experimental results given above.

The theoretical prediction of the change of particle concentration is obtained by solving Eq. (4) by a procedure described elsewhere, ${ }^{5}$ ) assuming 200 -fold particles are the largest. Dashed lines in the succeeding figures except Fig. 10 indicate the regions where the error in total volume, of particles, due to the appearance of over 200 -fold particles, exceeds $5 \%$.

Experimental results are, first of all, compared with the Saffman-Turner theory in which $\alpha_{T}$ in Eq. (4) is unity, assuming that the effective energy dissipation $\varepsilon$ is given by $\varepsilon_{0}$ defined by Eq. (5). By taking a dimensionless time $t^{+}\left(\equiv 1.294(\varepsilon / v)^{1 / 2} a_{1}{ }^{3} N_{0} t\right)$ as the abscissa, the theoretical value of $N_{t} / N_{0}$ is given by a single curve independently of $N_{0}, \varepsilon$ and $a_{1}$. Figure 7 is the replot of the data in Fig. 2 against $t^{+}$. It is found that the data for $N_{0}$ greater than $4.08 \times 10^{7} \mathrm{~cm}^{-3}$, which is called critical concentration $N_{c}$, fall on a single curve as predicted theoretically, while the data of $N_{0}<N_{c}$ deviate from the curve with decreasing of

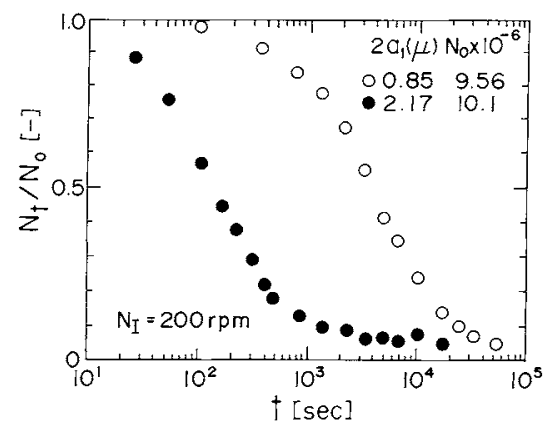

Fig. 6. Change of $N_{t} / N_{0}$ for particles of different sizes.

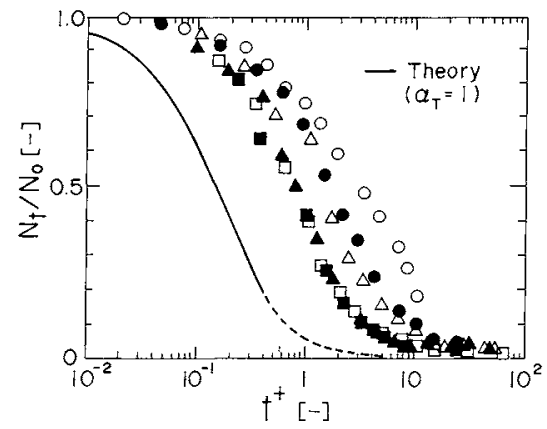

Fig. 7. Comparison of data in Fig. 2 with Saffman-Turner theory. (Key for symbols is as in Fig. 2.)

$N_{0}$. A similar feature is also found for dispersions of $2.17 \mu \mathrm{m}$ particles for which $N_{c}$ is equal to $1.26 \times 10^{6}$ $\mathrm{cm}^{-3}$. No theories proposed so far can explain this deviation. It is possibly due to the failure of assumption (1) given in $\S 1$, because $R_{i j}$ becomes comparable with the eddy size of turbulence as $N_{0}$ decreases. But more detailed experiments are required to clarify the reason completely. We leave this problem to further study, and the data of $N_{0} \geqq N_{c}$ are employed in the following arguments. Figure 7 indicates that the relation $N_{t} / N_{0}$ vs. $t$ is essentially the same in shape between theory and experiment but differs greatly in magnitude.

The data in Fig. 4 are replotted against $t^{+}$in Fig. 8. It appears as if all the data fall on a single curve independently of $N_{I}$ within experimental error. However, these curves shift from left to right with increasing of $N_{I}$ when watched carefully. This shift, which is also found for dispersions of $2.17 \mu \mathrm{m}$ particles, cannot be explained by the Saffman-Turner theory but explained by the present theory, as discussed later. Figure 9 shows the replot of $N_{t} / N_{0}$ in Fig. 6 against $t^{+}$. Here the data of $N_{0}=4.08 \times 10^{7} \mathrm{~cm}^{-3}$ are used instead of those of $N_{0}=9.56 \times 10^{6} \mathrm{~cm}^{-3}$ for $0.85 \mu \mathrm{m}$ particles, because the latter concentration is less than $N_{c}$ as shown in Fig. 7. The dependence of $N_{t} / N_{0}$ on particle size predicted by the theory is satisfactory. The comparison made in these figures implies that the Saffman-Turner theory overestimates the magnitude of coagulation rate too much to use in estimating the 


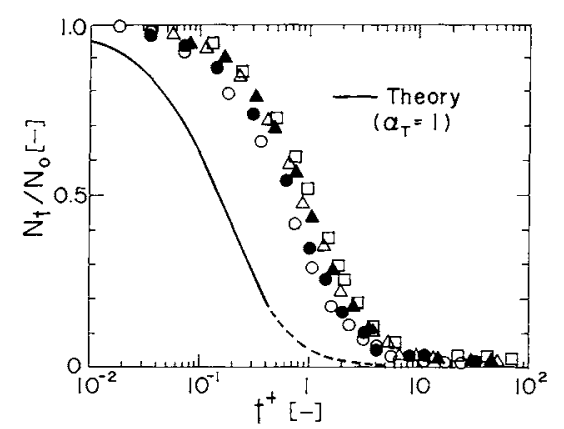

Fig. 8. Comparison of data in Fig. 4 with Saffman-Turner theory. (Key for symbols is as in Fig. 4.)

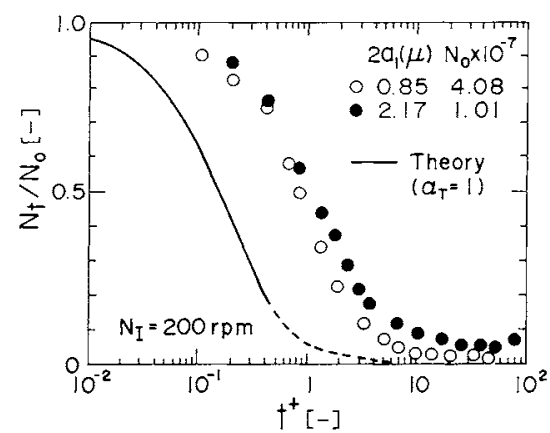

Fig. 9. Comparison of data of $N_{t} / N_{0}$ change for particles of different sizes with Saffman-Turner theory.

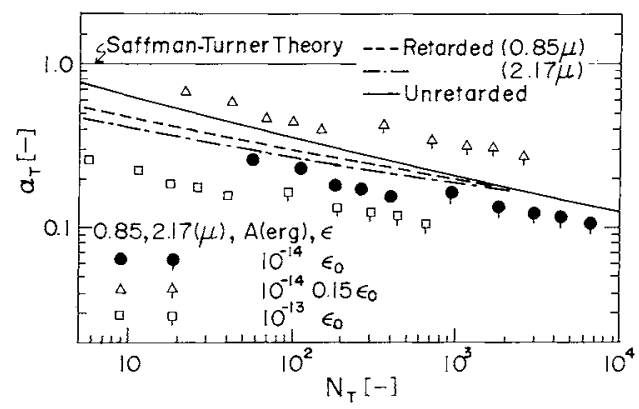

Fig. 10. Theoretical and experimental values of $\alpha_{T}$.

process of turbulent coagulation of aqueous dispersions in a stirred tank.

Now the experimental results are compared with prediction by the present theory. Relations between $\alpha_{T}$ and $N_{T}\left(=6 \pi \mu a_{1}^{3} \gamma / A\right)$, obtained by repeating a manipulation described elsewhere, ${ }^{5)}$ are drawn in Fig. 10 for the retarded and unretarded van der WaalsLondon interparticle potentials. Assuming that (i) the Hamaker constant $A$ for latex particles is $10^{-1.4} \mathrm{erg}$, (ii) the interparticle force is given by the retarded potential and (iii) the deformation rate $\dot{\gamma}\left(=\overline{\left.d U_{x} / d y\right)}\right.$ is given by $\dot{\gamma}=(4 \varepsilon / 15 \pi v)^{1 / 2},{ }^{11,13)}$ the theoretical values of $\alpha_{T}$ for these dispersions are obtainable from Fig. 10. Substituting $\alpha_{T}$ into $t^{*}$, the data in Figs. 2, 4 and 6 are replotted against $t^{*}$ in Figs. 11, 12 and 13 respectively. It is clear the agreement between theory and experiment is improved considerably; that is, the difference in magnitude of $N_{t} / N_{0}$ is reduced and the shift of the

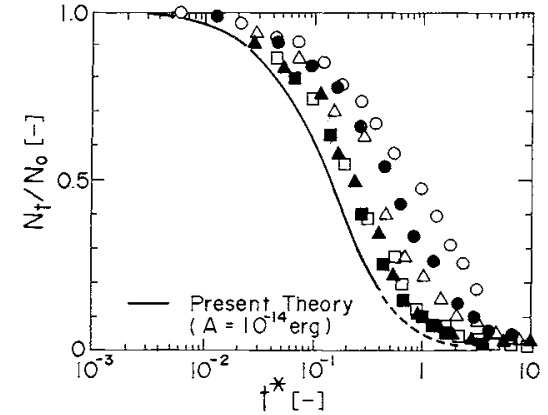

Fig. 11. Comparison of data in Fig. 2 with present theory (Key for symbols is as in Fig. 2.)

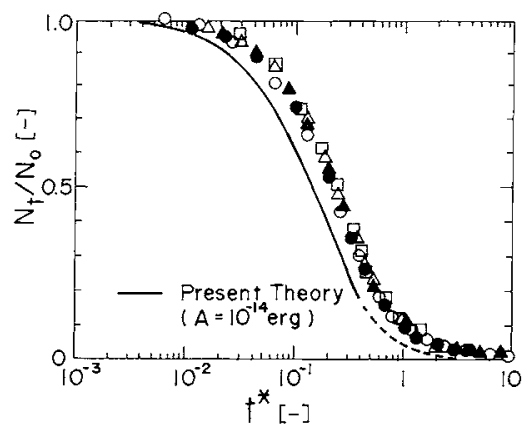

Fig. 12. Comparison of data in Fig. 4 with present theory. (Key for symbols is as in Fig. 4.)

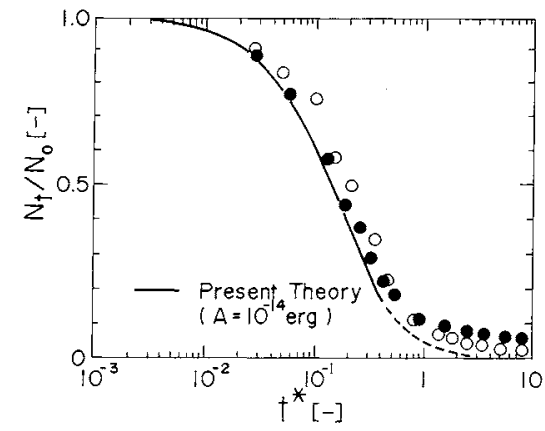

Fig. 13. Comparison of data in Fig. 9 with present theory. (Key for symbols is as in Fig. 9.)

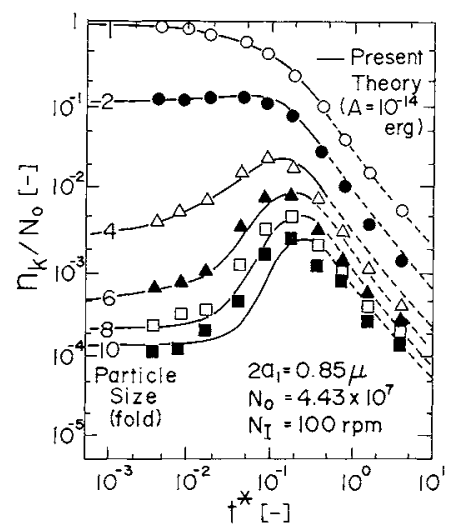

Fig. 14. Comparison of concentration change of particles of each size with theoretical prediction.

relation $N_{t} / N_{0}$ vs. $t$ in Fig. 8 disappears in Fig. 12. Figure 14 shows a typical change of concentration of particles of each size. It is confirmed that prediction 
by this theory is satisfactory, especially in the case of small particles.

Experimental values of $\alpha_{T}$ may be evaluated by fitting Eq. (4) to the data of $N_{t} / N_{0} \geqq 0.2$ in Fig. 4 and the data for $2.17 \mu \mathrm{m}$ particles, such that the sum of squared errors is minimized by the Simplex method. As expected from the good coincidence in Fig. 12, the dependence of $\alpha_{T}$ on $N_{T}$ coincides fairly well with the theoretical relation as shown in Fig. 10. In this treatment it is assumed that $A$ is $10^{-14} \mathrm{erg}$ and that the power input to the impeller is dissipated completely in the bulk of the tank. However, it is known that the definite value of $A$ for latex particles is uncertain but is somewhere between $10^{-14}$ and $10^{-13}$ erg, ${ }^{8)}$ and that some of the power input may be dissipated in the impeller or at the wall surface. It is difficult to estimate what proportion of the input energy is dissipated as the effective energy. Investigations on the turbulence in a stirred $\operatorname{tank}^{3,4,14,15)}$ imply that the value of $\varepsilon$ is somewhere between $0.15 \varepsilon_{0}$ and $\varepsilon_{0}$. Experimental values of $\alpha_{T}$ for various combinations of the extreme values of $A$ and $\varepsilon$ are shown in Fig. 10. It is important to find that they are placed around the theoretical curve and that the slope of the relation $\alpha_{T}$ vs. $N_{T}$ is similar between theory and experiment. This means that $\alpha_{T}$ in the coagulation of common aqueous dispersions will also be placed around the theoretical curve. On the other hand, it is clear that the Saffman-Turner theory yields a considerable error even when $\varepsilon=0.15 \varepsilon_{0}$ is assumed.

These results suggest that it is necessary to introduce the hydrodynamic interaction between particles into the kinetic equation. It is important to note that this conclusion is consistent with that drawn previously for the Brownian coagulation. ${ }^{6)}$

\section{Conclusions}

1) A kinetic equation of turbulent coagulation for dispersions of colloidal particles in a viscous fluid in which the hydrodynamic interaction between particles is taken into account is formulated.

2) Changes of particle concentration for the coagulation of latex particles in a stirred tank are successfully explained by the newly proposed theory.

3) The Saffman-Turner theory overestimates the coagulation rate considerably and yields a systematic error in the dependence of coagulation rate on energy dissipation when the theory is applied to the turbulent coagulation of common aqueous dispersions in a stirred tank.

\section{Nomenclature}

$A$

$=$ Hamaker constant

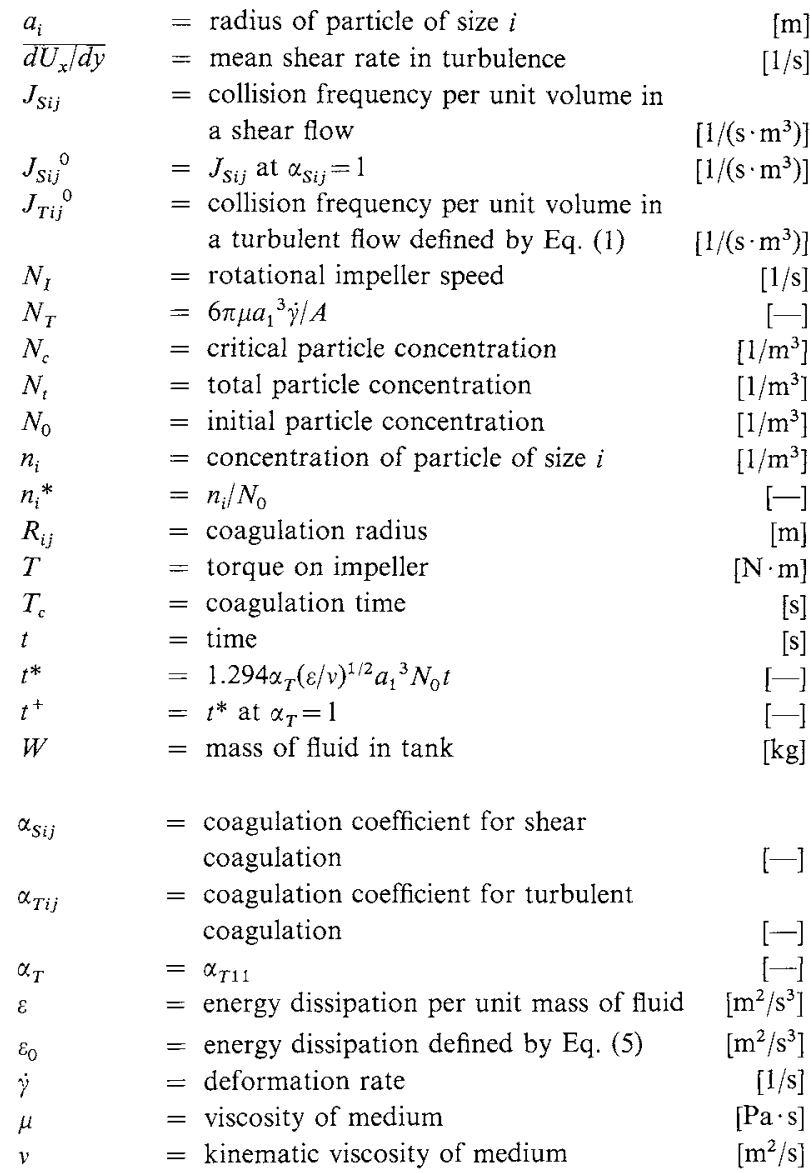

〈Subscript〉

$i j=$ subscript indicating correlation between particles of sizes $i$ and $j$

\section{Literature Cited}

1) Beal, S. K.: Aerosol Sci., 3, 111 (1972).

2) Camp, T. R. and P. C. Stein: J. Boston Soc. Civ. Eng., 30, 219 (1943).

3) Cutter, L. A.: AIChE J., 12, 35 (1966).

4) Günkel, A. A. and M. E. Weber: AIChE J., 21, 931 (1975).

5) Higashitani, K., R. Ogawa, G. Hosokawa and Y. Matsuno: $J$. Chem. Eng. Japan, 15, 299 (1982).

6) Higashitani, K. and Y. Matsuno: J. Chem. Eng. Japan, 12, 460 (1979).

7) Levich, V. G.: "Physicochemical Hydrodynamics," p. 213, Prentice Hall, London (1962).

8) Lyklema, J.: Advan. Colloid Interface Sci., 2, 65 (1968).

9) Nishikawa, M., Y. Okamoto, K. Hashimoto and S. Nagata: $J$. Chem. Eng. Japan, 9, 489 (1976).

10) Okuyama, K., Y. Kousaka, Y. Kida and T. Yoshida: J. Chem. Eng. Japan, 10, 142 (1977).

11) Saffman, P. G. and J. S. Turner: J. Fluid Mech., 1, 16 (1956).

12) Smoluchowski, M.: Z. Physik. Chem., 92, 129 (1917).

13) Takahashi, K.: "Kiso Aerosol Kogaku," p. 75, Yokendo, Tokyo (1972).

14) Tambo, N., K. Yamada and H. Hozumi: Suidokyokai Zasshi, 427, 4 (1970).

15) Toyama, S., T. Ameno and M. Sogame: Kagaku Kogaku Ronbunshu, 7, 524 (1981). 